\title{
Parameter identification and contact modeling for planetary wheeled rovers in soft soil
}

\author{
Alexandre C. Leite* Alberto Gallina* Bernd Schäfer* \\ * Deutsches Zentrum für Luft- und Raumfahrt, Institute of Robotics \\ and Mechatronics, Weßling, 82205, Germany (e-mail: \\ alexandre.carvalholeite@dlr.de, alberto.gallina@dlr.de, \\ bernd.schaefer@dlr.de).
}

\begin{abstract}
A challenging topic in the field of planetary rover simulations is the modeling of the contact between the rover and its environment. Multibody simulation models are used to represent the kinematic structure of a wheeled rover, but its interface with the environment relies on sand-wheel contact dynamics. A new advanced contact model is presented and tuned according to single wheel experiments by comparison between experimental data and predicted values. Finally, stochastic model updating is performed to assess parameter uncertainty level due to soil properties. As a result, the mean percentage error of the tuned model is inside the acceptable range, but additional improvements in the rover sinkage computation model are still desired.
\end{abstract}

Keywords: parameter identification, parameter estimation, modelling errors, dynamic modelling, space vehicles

\section{INTRODUCTION}

The dynamic model to identify is the contact model between a single wheel and deformable terrain. It is a breadboard version of a planetary exploration rover intended to operate on Mars and drive on a rocky environment with loose sandy soil. Our modeling efforts allow simulation of a rover in different scenarios containing complexly shaped rocks and undulating terrain with different kinds of soft soil and rigid surfaces. The contact between wheels and soft soil is of major concern due to critical constraints (i.e. excessive slip and sinkage) imposed to the mobility of the vehicle in such terrains. The following section describe the contact modeling for a smooth rigid wheel in soft soil and the contact dynamics of the propelling grousers which can be attached to the smooth wheels to improve the traction performance in soft soil.

\section{MODELING}

\subsection{Contact dynamics of a smooth wheel in soft soil}

Our model must be able to predict contact forces and reaction torques when interfacing the vehicle with the terrain through rigid wheels. These wheels can either operate in towed mode or actuated mode with driven by motors, and steerable or not steerable; which allow us to define the forces to be modeled to cope with each one of these situations.

Towed wheels are not able to produce traction but are subjected to the motion resistance forces as the rover moves and sand is compacted (compaction) and pushed (bulldozing). Driven wheels produce traction as a function of the available soil thrust. Steering maneuvers are affected by longitudinal forces and lateral forces as well. Lateral forces will not be considered in this paper, because our tests were performed without steering maneuvers. Compaction and traction are the most dominant longitudinal forces and are modeled as follows.

Figure 1-left shows a driven smooth rigid wheel sank in soft soil; figure 1-right shows a diagram of the forces and stresses considered. The wheel has radius $r$, width $b$ and is subjected to the load $F_{L}$ (weight of the kinematic structure of the rover plus payload, sensors, motor, batteries, etc. are lumped into this force). The contact region with the soil is defined with respect to the polar coordinates fixed in the wheel from $\theta_{r}$ (rear part) to $\theta_{f}$ (front part); which are respectively functions of $\lambda z$ and $z$, see figure 1 where $\lambda$ is the sinkage ratio. The normal pressure distributed under the rim is $\sigma(\theta)$, where $\theta$ is the same polar coordinate fixed in the wheel. $\tau(\theta)$ is the shear stress which generates traction. Drawbar pull is the resulting force $F_{D B}=F_{T}-$ $F_{C}$, where $F_{T}$ is the traction force and $F_{C}$ the compaction force. Both forces can be obtained integrating shear stress and normal pressure distribution in the direction of motion as follows

$$
F_{D B}=r b \int_{\theta_{f}}^{\theta_{r}}(\tau \cos \theta-\sigma \sin \theta) \mathrm{d} \theta
$$

Accordingly with Bekker (1956) the resistance torque is obtained by the integral of the shear stresses.

$$
T=r^{2} b \int_{\theta_{f}}^{\theta_{r}} \tau \mathrm{d} \theta
$$




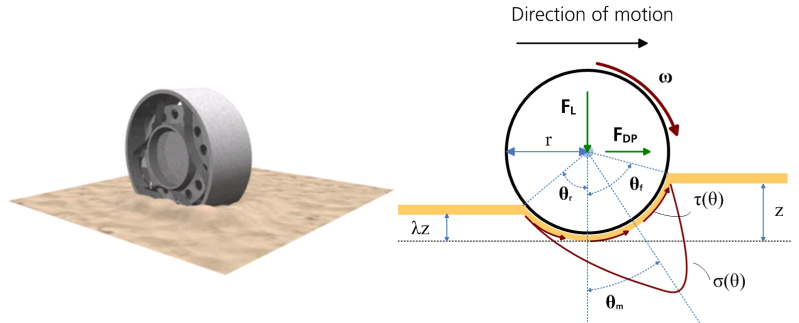

Fig. 1. (Left) perspective of the modelled situation. (Right) stresses and forces in the contact between a loaded wheel and sand

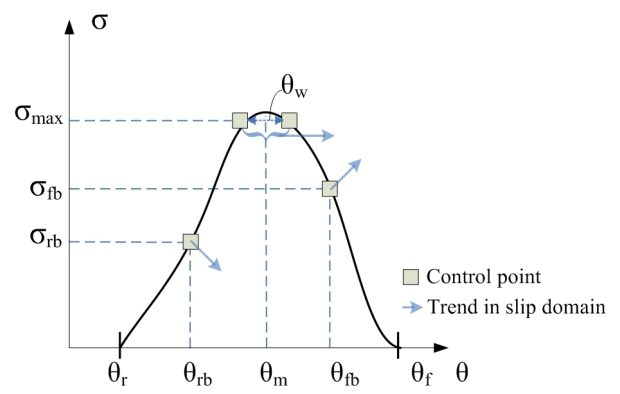

Fig. 2. Distribution of the normal pressure under the wheel

The shear stress is a function of the pressure under the wheel, $\tau=(c+\sigma \tan \phi)\left[1-e^{\frac{-j}{k}}\right]$. Cohesion $c$ and friction angle $\phi$ are mechanical properties of the soil considered as known a-priori. Soil deformation $j$ and shear deformation modulus $k$ describe the shear stress deformation curve. In Wong (2001) the soil deformation is derived as a function of slip ratio: $j=r\left[\theta_{f}-\theta-(1-s)\left(\sin \theta_{f}-\sin \theta\right)\right]$. A positive slip ratio $s=1-\frac{v}{r \omega}$ holds for $r \omega>v$, i.e. the wheel rotating faster than the achieved translational velocity in the longitudinal direction. The value of $k$ is a parameter to be estimated. Also $\sigma$ has to be estimated, but this is a function of $\theta$ and not a parameter. There are classical analytic descriptions of $\sigma$ which are normally used in the literature Shilby et al. (2005), but these are empirical models unable to model subtleties concerning the change of its shape as a function of slip ratio and load.

Our numerical description of $\sigma$ is based on Chaikin (1974) for smooth and fast computation of the curve in figure 2 by four control points. This method generates a quadratic uniform B-spline curve and is very useful because allows to adjust the curve by successive refinement of the given control polygon. The control points (which generate a control polygon) are defined by the numerical values of $\theta_{r b}$, $\theta_{m}, \theta_{f b}, \theta_{w}, \sigma_{r b}$, and $\sigma_{f b}$. But there are some constraints to these parameters in order to get meaningful results comparing with the experimental knowledge available in the terramechanics literature. Note that $\theta_{r}$ and $\theta_{f}$ are not freely changed, they depend on sinkage $z$ and sinkage ratio $\lambda$ of the rear part of the wheel. The maximal normal pressure is given by Bekker's equation $\sigma_{\max }=\left(\frac{k_{c}}{b_{s}}+k_{\phi}\right) z^{n}$, considering $b_{s}$ as the smaller dimension of the rectangular patch formed by the wheel sunk into the soil. For small sinkage depths $b_{s}<b$ is considered. The sinkage $z$ and the smaller dimension of the contact patch $b_{s}$ are computed through the inversion of the following equation

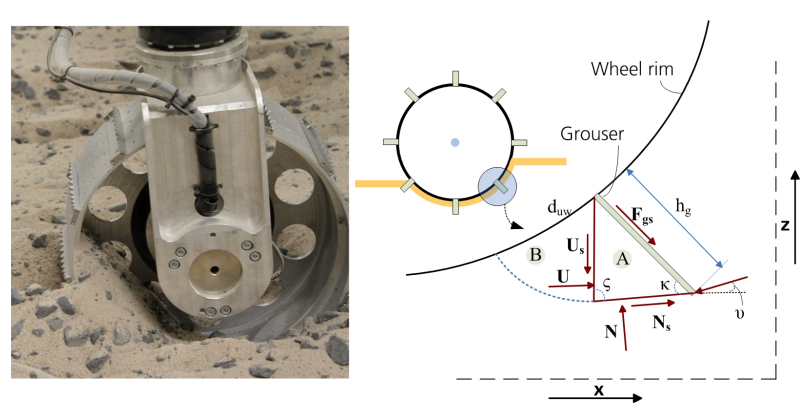

Fig. 3. (Left) photo of the experimental situation as reference for the modeled case. (Right) contact forces between grouser and failure regions

$$
F_{N}=r b \int_{\theta_{f}}^{\theta_{r}} \sigma \cos \theta \mathrm{d} \theta
$$

A search procedure is implemented to find the values of $b_{s}$ and $z$ which always agree with equation 3. Equations 1-3 allow the computation of the variables to be compared with the measured experimental data (drawbar pull, torque, sinkage), the parameter vector $q_{s}, q_{s}=$ $\left[\lambda k \theta_{r b} \theta_{m} \theta_{f b} \theta_{w} \sigma_{f b} \sigma_{r b}\right]^{T}$ has to be estimated in order to achieve an acceptable model correlation at each slip value.

\subsection{Contact dynamics of grousers in contact with sand}

Grousers can be attached to a smooth wheel to improve its driving performance, especially weak terrain. Figure 3 shows our rigid wheel with grousers and the scheme of the corresponding contact geometry at one grouser.

There are two failure regions illustrated in the scheme of figure 3, they are the equilibrium wedge $\mathbf{A}$ and the $\log$ spiral zone $\mathbf{B}$ as described in Hermawan et al. (2000). In two dimensions, the traction force of the grouser is

$$
F_{t g}=F_{g s}+U+U_{s}+N+N_{s}
$$

where $F_{g s}$ is the force on the interface region $\mathbf{A}$-grouser interface, $U$ on the region $\mathbf{B}, U_{s}$ due to cohesion on the interface A-B, $N$ gravitational force of $\mathbf{A}, N_{s}$ due to cohesion on the interface region $\mathbf{A}$-soil under $\mathbf{A}$. The drawbar pull of equation 1 can be revised by adding the term $F_{t g} b$, the multiplication by $b$ is to correct for a three dimensional wheel driving straight ahead without camber angle. The equilibrium wedge is a function of the terrain properties and specific interaction between soil and grouser, since $\varsigma=90-\phi$ and $\kappa=f(v, \alpha)$ ( $\alpha$ is the rotation angle of the wheel) define two dimensions of the equilibrium wedge. The angle $v$ is the instantaneous direction in which the soil is moving. A vector with components in directions $x$ and $z$ is the tractive force $F_{t g}$ provided by the grouser to lift and push the wheel. In the present analysis just the pushing component is important, since the lifting force is very small compared with the weight of the wheel plus load.

Three additional parameters have to be estimated, they describe 1) the movement of sand at the basis of the equilibrium wedge and 2) the amount of sand pushed to the back of the wheel. The first set of parameters is defined as a linear dependence on the angle of rotation of the 
wheel: $v=\xi_{1} \alpha+\xi_{2}$. The second set is defined as a slip dependent additive sinkage to the rear part of the wheel: $\lambda_{1} s z$. Another parameter vector to be estimated can be constructed as

$$
q_{g}=\left[\begin{array}{lll}
\xi_{1} & \xi_{2} & \lambda_{1}
\end{array}\right]^{T}
$$

\subsection{Normal pressure distribution}

The normal pressure distribution $\sigma$ is a particularly important function, all variables to be identified are strongly dependent on this function. The classical description of $\sigma$ is based on the pressure-sinkage relationship for a rectangular plate, a wheel is described like small plates vertically pushed against the soil in different sinkage depths. Some practical works like Hegedus (1962) showed that the shape of the normal pressure distribution is not so well behaved like the classical analytical description predicts. Nevertheless, we know that the amplitude of the maximal pressure is fairly accurate and that the shape of this function has some trend in the slip domain. The arrows in figure 2 illustrate the trend of the shape as the slip ratio increases. These assumptions allow us to define the constrains to $\sigma$ parameters listed in table 1

Table 1. Constraints to the trend of $\sigma$ in the slip domain

\begin{tabular}{cc} 
Parameter & Monotonic trend \\
\hline$\theta_{r b}$ & non decreasing \\
$\theta_{m}$ & non decreasing \\
$\theta_{f b}$ & non decreasing \\
$\theta_{w}$ & non increasing \\
$\sigma_{r b}$ & non increasing \\
$\sigma_{f b}$ & non decreasing \\
\hline
\end{tabular}

However, there is an infinity number of functions that describe those monotonic trends. As solution, we consider that the changes at $\sigma(\theta)$ are smooth with $s$ by introducing the following functions

$$
\begin{gathered}
\rho_{n d}(s)=\left\{\begin{array}{l}
\left(1-\rho_{n d}(0)\right)\left(1-e^{-s / \rho_{n d}^{D}}\right)+\rho_{n d}(0): \rho_{n d}^{D}>0 \\
\left(1-\rho_{n d}(0)\right)\left(e^{\rho_{n d}^{D} / s+\rho_{n d}^{D}}\right)+\rho_{n d}(0): \rho_{n d}^{D}<0
\end{array}\right. \\
\rho_{n i}(s)= \begin{cases}-\rho_{n i}(0) e^{-s / \rho_{n i}^{D}} & : \rho_{n i}^{D}>0 \\
-\rho_{n i}(0)\left(1-e^{\rho_{n i}^{D} / s+\rho_{n i}^{D}}\right) & : \rho_{n i}^{D}<0\end{cases}
\end{gathered}
$$

were the subscripts ${ }_{n i}$ and ${ }_{n d}$ stand for non increasing and non decreasing respectively. These monotonic smooth functions were chosen because they are able to represent almost linear behavior and smooth abrupt changes as well. The parameters $\rho^{D}$ can be substituted by the corresponding parameters in table 1 to make the following parameter vector to be estimated

$$
q_{p}=\left[\begin{array}{llllll}
\theta_{r b}^{n d} & \theta_{m}^{n d} & \theta_{f b}^{n d} & \theta_{w}^{n i} & \sigma_{r b}^{n i} & \sigma_{f b}^{n d}
\end{array}\right]^{T}
$$

It results in slip varying control points of the $\sigma$ curve like $\theta_{r b}=f\left(\theta_{r b}, \theta_{r b}^{n d}, s\right), \ldots, \sigma_{f b}=f\left(\sigma_{f b}, \sigma_{f b}^{n d}, s\right)$.

\section{IDENTIFICATION METHOD AND TOOL}

For identification and optimization purposes we have an in-house developed tool, MOPS (Multi-Objective Parameter Synthesis), implemented as a Matlab toolbox. In this

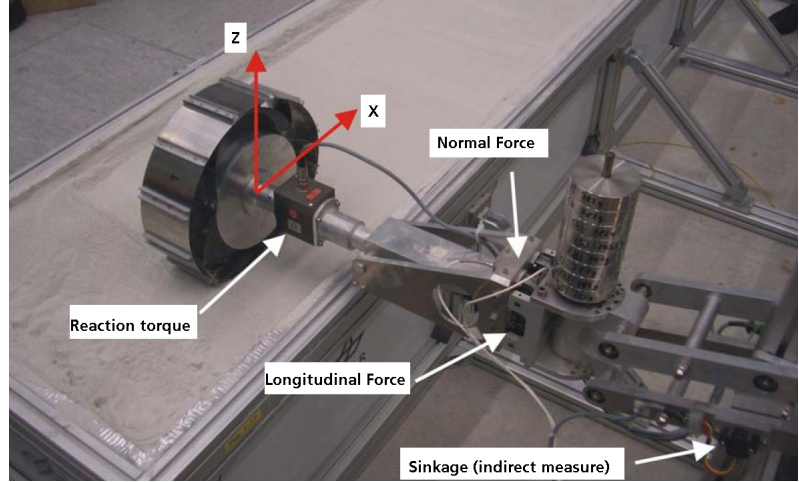

Fig. 4. Single wheel test: experiment setup

tool we are able to define the parameters to be estimated and the objective functions to be minimized. As a normalization factor the value of $20 \%$ of MPE (mean percentage error for $n$ samples of index si, $M P E=$ $\left.\frac{1}{n} \sum_{s i=1}^{n} \frac{\text { predicted }_{s i}-\text { measured }_{s i}}{\text { measured }_{s i}}\right)$ between the computed values of $F_{D P}, T$ and $z$ from equations $1-2$ and the measured data is used. We intend to minimize the objective function $J=\frac{1}{2}\left(\frac{F_{D P, M P E}}{20}+{\frac{T_{M P E}}{20}}^{2}+\frac{z_{M P E}}{20}\right)$ by changing the parameter vector $q=\left[\begin{array}{lll}q_{s} & q_{g} & q_{p}\end{array}\right]$. The optimization algorithm chosen was DE (differential evolution) in order to perform global minimization over the continuous space of $q$ considering nonlinear changing of the objective function. DE was implemented like in Storn and Price (1995), and the strategy to generate candidates $c$ for new population members was

$$
c^{i}=q^{i}+F B \cdot\left(q^{b e s t}-q^{i}\right)+F \cdot\left(q^{r 1}-q^{r 2}\right)
$$

where $c^{i}$ is the new candidate, $q^{i}$ the current parameter vector, $F B=0.8$ the amplification factor, $F=0.8$ the amplification factor for random differential variation, and the indices $r_{1}$ and $r_{2}$ are integers randomly chosen from the interval $[1, P] \in \mathbb{Z}, P$ is the population size normally chosen as two times the size of vector $q$.

\section{SINGLE WHEEL TESTS}

Several drawbar pull tests were performed at the Single Wheel Testbed (SWT) of the DLR's Institute for Space Systems (DLR-RY), see figure 4.

A drawbar pull test consists of imposing a translational velocity to the wheel and commanding its nominal angular velocity so that a known slip ratio is obtained. The tests covered a range from $0 \%$ to $90 \%$ slip at three different normal load cases. Each drawbar pull, torque and sinkage measurement set was used to tune the model described in section 2 using the method and tool introduced in section 3 . The tuned model shows that the pressure distribution under the wheel changes its shape as a function of load and slip as figure 5 illustrates.

Figure 5-top shows a comparison between the equivalent analytic classical description and the identified shape. Figure 5-bottom shows the shape at the three load cases. Figures 6,7 , and 8 show the correlation between measured and predicted forces/torques for the 3 load cases. This pressure distribution was assumed as the same for smooth wheel and for a lugged wheel 

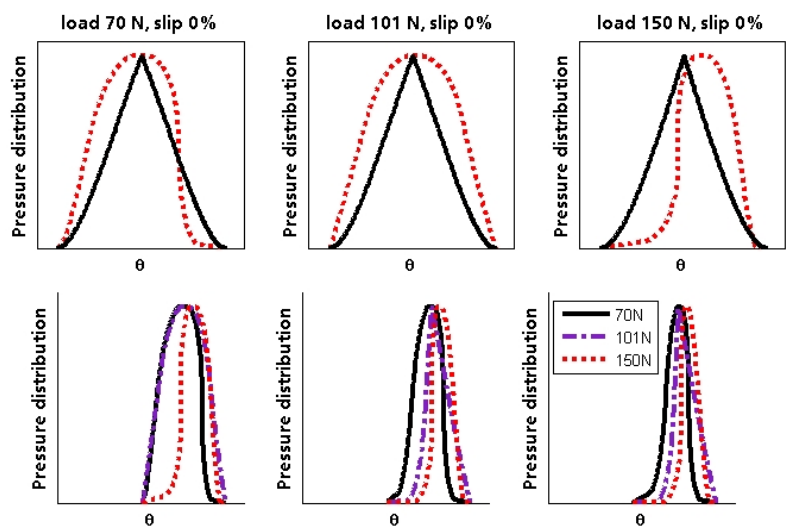

Fig. 5. Top: pressure distribution at $0 \%$ slip, (solid) classical and (dotted) numeric description. Bottom: three different slip values, $0 \%$ (left), 50\% (middle) and 90\% (right)
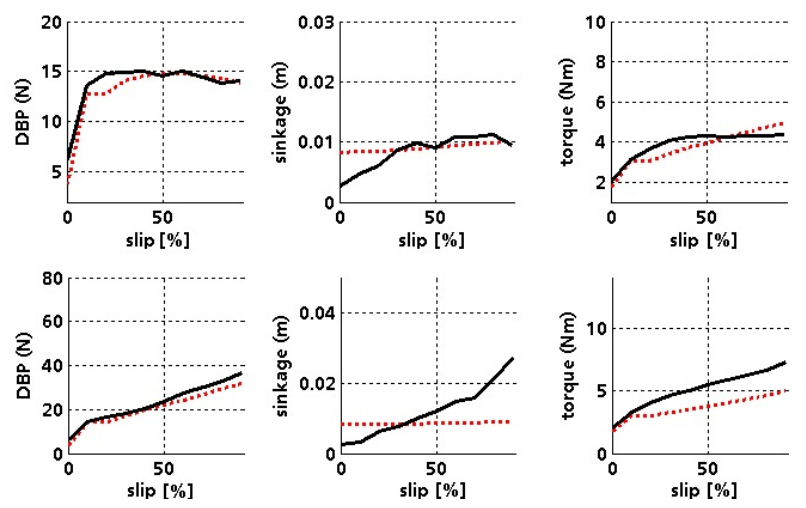

Fig. 6. Load case 70N: correlation of drawbar pull and torque in slip domain and sinkage constraint for smooth wheel (top) and lugged wheel (bottom)
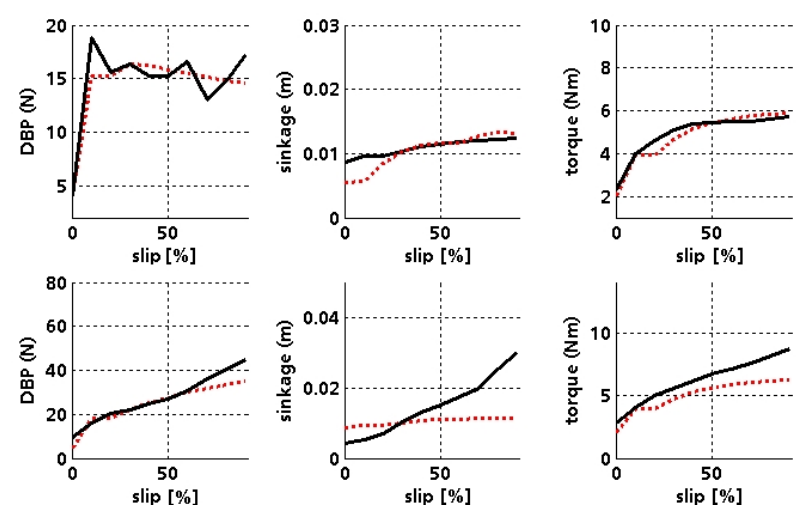

Fig. 7. Load case 101N: correlation of drawbar pull and torque in slip domain and sinkage constraint for smooth wheel (top) and lugged wheel (bottom)

The agreement of drawbar pull and resistance torque remains in the acceptable range $(20 \%)$, but the sinkage prediction does not fit very well to the measurement data. The prediction errors can be summarized as follows in table 2 .

A MPE of $20 \%$ is acceptable to our purposes. Although, incorporation of external effects present in the dynamics of the test apparatus and its interaction with the wheel
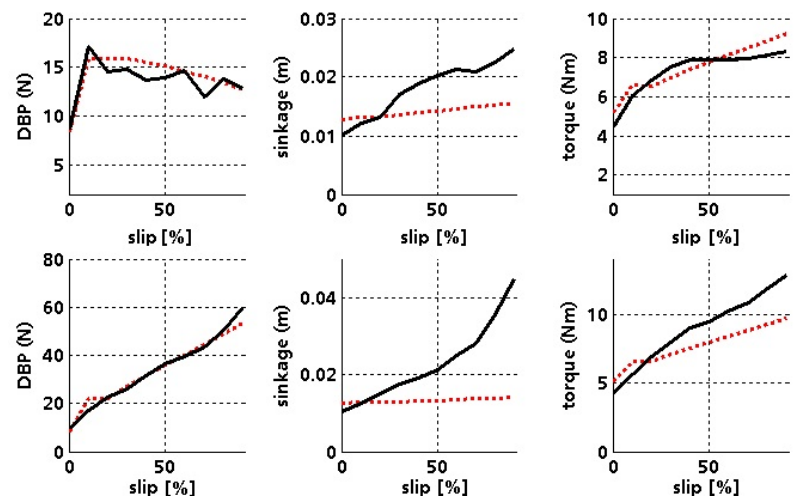

Fig. 8. Correlation of drawbar pull and torque in slip domain and sinkage constraint for smooth wheel (top, load case $150 \mathrm{~N}$ ) and lugged wheel (bottom, load case $147 \mathrm{~N})$

could improve the results. Currently, we do not know how friction and internal slip in the mechanisms are disturbing the experiment. Also the soil parameters (Bekker's parameters) are affected by variability which can disturb the results, this issue is discussed in the next section.

\section{STOCHASTIC MODEL UPDATING}

The parameter estimation of section 3 considers deterministic input and output parameters and searches for a model parameter setup that yields a good matching with experimental data. Nevertheless, model parameters are often affected by inherent variability that calls for a stochastic treatment of the problem. Experimental evidence, Apfelbeck et al. (2010), shows that the Bekker parameters measured via Bavameter tests are always affected by significant uncertainty. If this uncertainty is accounted for and propagated through the computer model, the predicted outputs become non-deterministic quantities as well. A more comprehensive updating procedure seeks parameter uncertainty levels that result in a satisfying agreement between the variability of given model outputs and the corresponding experimental variability.

Here, we consider a stochastic procedure where means and variances of the Bekker's parameters, modeled as uncorrelated normal random variables, are tuned. The corresponding parameter vector to estimate is

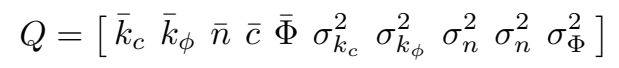

where $\boldsymbol{\bullet}$ and $\sigma_{\bullet}^{2}$ denote the mean and variance of $\bullet$.

The quantity to minimize is the squared loss function $L=F^{T} F$ where

$$
F=\left[\begin{array}{llllll}
\bar{F}_{1} & \bar{F}_{2} & \bar{F}_{3} & \sigma_{F_{1}}^{2} & \sigma_{F_{2}}^{2} & \sigma_{F_{3}}^{2}
\end{array}\right]
$$

Table 2. Mean percentage error between measurement data and model prediction for each set of tests

\begin{tabular}{c|ccc} 
Wheel type & Load case & DP error & Torque error \\
\hline \multirow{3}{*}{ Smooth } & $70 N$ & $7.6 \%$ & $9.8 \%$ \\
& $101 N$ & $7.0 \%$ & $5.4 \%$ \\
& $150 N$ & $7.0 \%$ & $7.5 \%$ \\
\hline \multirow{3}{*}{ Lugged } & $70 N$ & $12 \%$ & $26 \%$ \\
& $101 N$ & $13 \%$ & $19 \%$ \\
& $147 N$ & $6.0 \%$ & $17 \%$ \\
\hline
\end{tabular}


with

$$
\begin{array}{r}
\bar{F}_{i}=\left[\Delta \bar{f}_{i}\left(s_{1}\right) \cdots \Delta \bar{f}_{i}\left(s_{1} 0\right)\right] \\
\sigma_{F_{i}}^{2}=\left[\Delta \sigma_{f_{i}}^{2}\left(s_{1}\right) \cdots \Delta \sigma_{f_{i}}^{2}\left(s_{1} 0\right)\right]
\end{array}
$$

and

$$
\begin{array}{r}
\Delta \bar{f}_{i}\left(s_{j}\right)=\bar{f}_{i}^{e x}\left(s_{j}\right)-\bar{f}_{i}^{n u m}\left(s_{j}\right) \\
\Delta \sigma_{f_{i}}^{2}\left(s_{j}\right)=\sigma_{f_{i}}^{2}{ }^{e x}\left(s_{j}\right)-{\sigma_{f_{i}}^{2}}^{\text {num }}\left(s_{j}\right)
\end{array}
$$

where $f_{1}=F_{D B P}, f_{2}=T, f_{3}=z$ and $\bullet^{e x}\left(s_{j}\right)$ and - ${ }^{\text {num }}\left(s_{j}\right)$ denote the experimental and numerical value of the quantity $\bullet$ calculated at the slip value $s_{j}$, with $j=1, \ldots, 10$ representing the analyzed 10 slip values The minimization of the loss function is a nonlinear least-squares problem which is solved through the 'trustregion-reflective' algorithm implemented in the standard optimization toolbox of Matlab. The algorithm requires at each step the determination of the loss function $L$, that is $F$, and the Jacobian matrix $J$ of $F$. The output parameter vector $F$ is estimated via crude Monte Carlo simulation (MCS), while the construction of $J$ implies the calculation of the Jacobian of means $J^{m}$ and variances $J^{v}$. A generic element of the matrix $J^{m}$ is defined as

$$
J_{l, k}^{m}=\left.\frac{\partial f_{i}\left(s_{j}\right)}{\partial p_{k}}\right|_{p_{k}=\bar{p}_{k}}
$$

with $l$ being function of $i$ and $j$ and $p_{k}$ the $k$-th model parameter of the set $\left[k_{c}, k_{p h i}, n, c, \Phi\right] . J_{l, k}^{m}$ is determined via finite differences at the current mean value of the parameter $p_{k}$. Under admissible assumptions Govers and Link (2010) the elements of $J^{v}$ can be approximated by

$$
J_{l, k}^{v}=-\left(J_{i j, k}^{m}\right)^{2}
$$

The optimization procedure starts assuming an initial parameter vector $Q^{(i n)}$ that defines the statistical parameters for the 100-sample MCS and the point at which the derivatives are to be evaluated. Once MCS is completed $F$ and $J$ are calculated as described above. Then, the trust-region-reflective algorithm identifies a new $Q^{(n e w)}$ and the procedure repeats until a stopping criterion is met. A general scheme of the optimization procedure is given in Figure 5. A more detailed description of the procedure can be found in Govers and Link (2010).

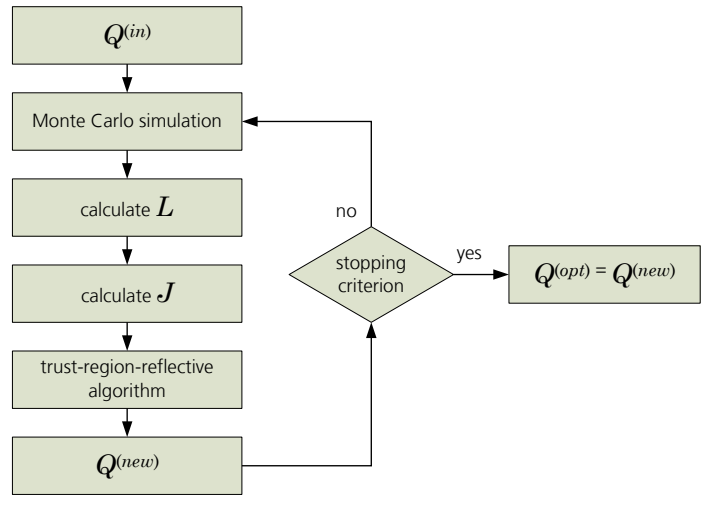

Fig. 9. Stochastic model updating procedure diagram
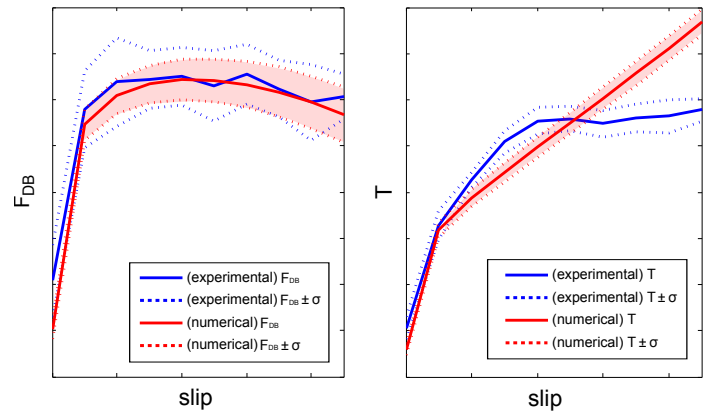

Fig. 10. Description of the $F_{D B}$ and $T$ variability after stochastic model updating procedure

In this application the mean terms of $Q^{(i n)}$ have been choosed equal to the optimal values obtained from the deterministic optimization, while the variance terms have been set to zero. After a few iterations the procedure converges to a solution that represents a local minimum for the loss function. The results of the stochastic model updating, presented in Table 5 and Figure 5, show almost no changes for the experimental-numerical correlation of the means of $F_{D B}, T$ and $z$ when compared with the results of the deterministic optimization (see Figure 6, load case $70 \mathrm{~N}$ ). However, large part of the experimental variance is now described by the variability of the Bekker's parameters.

Table 3. Initial and estimated statistics of the Bekker's parameters

\begin{tabular}{lrcrr} 
Parameter & $\bar{p}^{(i n)}$ & $\sigma_{p}^{(i n)}$ & $\bar{p}^{(o p t)}$ & \multicolumn{1}{c}{$\sigma_{p}^{(\text {opt })}$} \\
\hline$k_{c}\left[N / m^{n+1}\right]$ & $-6.67 \mathrm{e} 5$ & 0 & $-6.66 \mathrm{e} 5$ & $9.61 \mathrm{e} 4$ \\
$k_{p h i}\left[N / m^{n+2}\right]$ & $1.92 \mathrm{e} 8$ & 0 & $1.91 \mathrm{e} 8$ & $1.16 \mathrm{e} 7$ \\
$n[-]$ & 1.80 & 0 & 1.81 & $6.70 \mathrm{e}-3$ \\
$c[P a]$ & 13.00 & 0 & 14.87 & 4.28 \\
$\Phi[\mathrm{rad}]$ & 0.23 & 0 & 0.24 & $5.60 \mathrm{e}-3$ \\
\hline
\end{tabular}

\section{CONCLUSION AND OUTLOOK}

Modeling, parameter estimation and stochastic model updating were performed. The tuned model (with estimated parameters) includes the shape of the pressure distribution as a function of slip and load. The correlated curves show that the prediction of sinkage still have to be improved. Drawbar pull force and resistance torque fit to the experimental data with MPE smaller than $20 \%$, it can be further improved if external aspects are included in the modeling. Friction and slip between mechanical parts of the test apparatus could be estimated in future works to quantify or even decouple their influence from the tuned model. The contact model shall be used in the simulation of our full rover modeled as an articulated multibody system to finally validate the contact model against useful test cases. We are working on the validation of the model against the tests performed under the ExoMars B2X2 test campaign.

\section{ACKNOWLEDGEMENTS}

Parts of the work on contact modeling development was performed in conjunction with the HGF alliance project "Planetary Evolution and Life" (HGF - "HelmholtzGemeinschaft der Großforschungsanstalten"). And parts 
of the work leading to the parameter estimation results have received funding from the European Community's Seventh Framework Programme (FP7/2007-2013) under Grant Agreement $\mathrm{n}^{o}$ 262744. The authors wish to gratefully acknowledge the support by HGF and EC.

\section{REFERENCES}

Apfelbeck, A., Kuß, S., Rebele, B., and Schäfer, B. (2010). A systematic approach to reliably characterize soils based on bevameter testing. In Proc. of The 9th Asia-Pacific ISTVS Conference and Annual Meeting of Japanese Society for Terramechanics. Sapporo, Japan.

Bekker, M.G. (1956). Theory of Land Locomotion. Univ. of Michigan Press, Ann Arbor, MI.

Chaikin, G. (1974). An algorithm for high speed curve generation. Computer Graphics and Image Processing, 3, 346349 .

Govers, Y. and Link, M. (2010). Stochastic model updating covariance matrix adjustment from uncertain experimental modal data. Mechanical Systems and Signal Processing, 24, 696-706.

Hegedus, E. (1962). A preliminary analysis of the force system acting on a rigid wheel. Technical Report AD0401805, U. S. Army Ordinance Tank-Automotive Command, Detroit 9, Michigan.

Hermawan, W., Yamazaki, M., and Oida, A. (2000). Theoretical analysis of soil reaction on a lug of the movable lug cage wheel. Journal of terramechanics, 37, 6586.

Shilby, H., Iagnemma, K., and Dubowsky, S. (2005). An equivalent soil mechanics formulation for rigid wheels in deformable terrain, with application to planetary exploration rovers. Journal of Terramechanics, 42, 113.

Storn, R. and Price, K. (1995). Differential evolution - a simple and efficient adaptive scheme for global optimization over continuous spaces. Technical Report TR-95012, ICSI - International Computer Science Institute.

Wong, J.Y. (2001). Theory of Ground Vehicles. Wiley, New York, third edition. 